\title{
Hyperon electromagnetic form factors in the timelike region
}

\author{
Johann Haidenbauer ${ }^{1}$, Ulf-G. Meißner ${ }^{2,1,3}$, Ling-Yun Dai ${ }^{4}$ \\ ${ }^{1}$ Institute for Advanced Simulation, \\ Institut für Kernphysik and Jülich Center for Hadron Physics, \\ Forschungszentrum Jülich, D-52425 Jülich, Germany \\ ${ }^{2}$ Helmholtz Institut für Strahlen- und Kernphysik and Bethe Center for Theoretical Physics, \\ Universität Bonn, D-53115 Bonn, Germany \\ ${ }^{3}$ Tbilisi State University, 0186 Tbilisi, Georgia \\ ${ }^{4}$ School of Physics and Electronics, \\ Hunan University, Changsha 410082, China
}

Electromagnetic form factors of hyperons $(\Lambda, \Sigma, \Xi)$ in the timelike region, accessible in the reaction $e^{+} e^{-} \rightarrow \bar{Y} Y$, are studied. The focus is on energies close to the reaction thresholds, where the properties of these form factors are significantly influenced by the interaction in the final $\bar{Y} Y$ system. This interaction is taken into account in the calculation, utilizing $\bar{Y} Y$ potential models that have been constructed by the Jülich group for the analysis of data from the reaction $\bar{p} p \rightarrow \bar{Y} Y$ in the past. The enhancement of the effective form factor for energies close to the threshold, seen in experiments of $e^{+} e^{-} \rightarrow \bar{\Lambda} \Lambda$ and $e^{+} e^{-} \rightarrow \bar{\Sigma}^{0} \Lambda$, is reproduced. With regard to the reactions $e^{+} e^{-} \rightarrow \bar{\Sigma}^{-} \Sigma^{+}, \bar{\Sigma}^{0} \Sigma^{0}, \bar{\Sigma}^{+} \Sigma^{-}$a delicate interplay between the three channels is observed in the results at low energies, caused by the $\bar{\Sigma} \Sigma$ interaction. Predictions for the electromagnetic form factors $G_{M}$ and $G_{E}$ in the timelike region are presented for the $\Lambda, \Sigma$, and $\Xi$ hyperons.

PACS numbers: 13.40.Gp, 13.66.Bc, 12.39.Pn 


\section{INTRODUCTION}

Our knowledge on the electromagnetic form factors (EMFFs) of the nucleon in the timelike region has significantly improved over the last years. Information on these quantities is accessible in the $\bar{p} p$ annihilation process $\bar{p} p \rightarrow e^{+} e^{-}$ [1, 2], and likewise in the reactions $e^{+} e^{-} \rightarrow \bar{p} p$ and $e^{+} e^{-} \rightarrow \bar{n} n$ from where most of the recent data emerged [3 -7$]$ and where experimental efforts continue [8, 9]. For a general overview, see the review [10]. Regarding the EMFFs of hyperons $(\Lambda, \Sigma, \Xi, \ldots)$ in the timelike region, the development is somewhat lagging behind. Nonetheless, while only a handful of experiments [11, 12] and theoretical studies [13-15] were available a few years ago, the situation has changed definitely in recent times with various experimental [16 23] and theoretical [24 33] activities. To some extent this is surprising given that the study of hyperons offers advantages in accessing more detailed information on the EMFFs. Specifically, the self-analyzing character of the weak decay of the hyperons can be exploited to determine also the polarization as well as spin-correlation parameters for reaction such as $e^{+} e^{-} \rightarrow \bar{\Lambda} \Lambda, e^{+} e^{-} \rightarrow \bar{\Sigma} \Sigma$, or even $e^{+} e^{-} \rightarrow \Xi \Xi$. Those observables allow one to determine not only bulk properties such as the reaction cross section or the (effective) form factor but also the relative magnitude of the two EMFFs $G_{E}$ and $G_{M}$ and even the relative phase between them [10, 34]. A first measurement of that kind has been reported recently by the BESIII collaboration for the reaction $e^{+} e^{-} \rightarrow \bar{\Lambda} \Lambda$ at $\sqrt{s}=2.396 \mathrm{GeV}[20]$.

In the present work we take that achievement as motivation to explore the electromagnetic form factors of other hyperons in the timelike region. A study at their properties is timely given that cross sections for the reactions $e^{+} e^{-} \rightarrow \bar{\Sigma}^{0} \Lambda$ [12], $e^{+} e^{-} \rightarrow \Sigma \Sigma$ [12, 17, 21], and $e^{+} e^{-} \rightarrow \bar{\Xi} \Xi 22$, 23] have become already available, although not yet in a quantity and quality comparable to $e^{+} e^{-} \rightarrow \bar{\Lambda} \Lambda$ [12, 19, 20]. Measuring spin-dependent observables and, thereby, determining the properties of the form factors $G_{M}$ and $G_{E}$ is the logical next step [35]. We study the reactions $e^{+} e^{-} \rightarrow \bar{Y} Y$ near their threshold where the essential input is the $\bar{Y} Y$ interaction in the final state. In particular, we provide predictions for the energy dependence of the reaction cross sections and also for the magnitude and relative phase of the form factors $G_{M}$ and $G_{E}$. The calculation is done in close analogy to our analysis of the reaction $e^{+} e^{-} \rightarrow \bar{p} p$ [36] where the interaction in the $\bar{p} p$ system has been taken into account rigorously. It turned out that, after including the $\bar{p} p$ final-state interaction (FSI), available data on the proton form factor but also differential cross sections and the ratio $\left|G_{E} / G_{M}\right|$ could be reproduced fairly well. The employed $\bar{N} N$ interaction had been derived within chiral effective field theory [37, 38] fitted to results of a $\bar{p} p$ partial-wave analysis [39]. We note in passing that this approach has been also successfully applied to the reaction $e^{+} e^{-} \rightarrow \bar{\Lambda}_{c} \Lambda_{c}$ [40 42 .

Unfortunately, with regard to the $\bar{Y} Y$ interaction we are not in such a comfortable situation as for $\bar{N} N$. Without the option for obtaining direct empirical information about the $\bar{Y} Y$ force, constraints for that interaction can be only inferred from studies of FSI effects in reactions such as $\bar{p} p \rightarrow \bar{Y} Y$ and/or the analysis of $\bar{Y} Y$ correlation functions measured in heavy-ion collisions [43]. By far the best investigated case is $\bar{\Lambda} \Lambda$, where the reaction $\bar{p} p \rightarrow \bar{\Lambda} \Lambda$ has been extensively measured in the PS185 experiment at LEAR and where data are available (from the reaction threshold up to about $2.4 \mathrm{GeV}$ ) for total and differential cross-sections, but also for spin-dependent observables [44 48], thanks to the aforementioned self-analyzing weak $\Lambda$ decay. The level of information is much less satisfactory for other channels like $\bar{p} p \rightarrow \bar{\Sigma}^{0} \Lambda$ and/or $\bar{p} p \rightarrow \bar{\Sigma} \Sigma[49$ 51] and, in particular, for $\bar{p} p \rightarrow \bar{\Xi}$.

In the present investigation we employ a phenomenological $\bar{Y} Y$ model that has been developed by the Jülich group for the analysis of those PS185 data in the past $52-56]$. In case of the $\bar{\Lambda} \Lambda$ interaction there are several variants which all describe the $\bar{p} p \rightarrow \bar{\Lambda} \Lambda$ data quite well [52 54]. Indeed, these variants have been already used by us for calculating the $e^{+} e^{-} \rightarrow \bar{\Lambda} \Lambda$ reaction [24] and yielded cross sections well in line with the data. Interestingly, it turned out that one of the interactions even reproduces roughly the BESIII results on the ratio and phase of the EMFFs [20]. The interactions utilized for the $\bar{\Sigma}^{0} \Lambda$ and $\bar{\Sigma} \Sigma$ channels were also tuned to the pertinent experimental information from PS185 [55]. However, due to the limited number of data, these interactions are certainly not well constrained. Nonetheless, we believe that it is instructive to explore the corresponding predictions at the present stage. They should be considered mainly as a guideline and as a stimulation for future experiments. Of course, ultimately (complete) measurements of the reactions $e^{+} e^{-} \rightarrow \bar{\Sigma}^{0} \Lambda$ and $e^{+} e^{-} \rightarrow \bar{\Sigma} \Sigma$ will provide new additional and rather stringent constraints on the $\bar{\Sigma}^{0} \Lambda$ and $\bar{\Sigma} \Sigma$ forces and, in turn, should be used to pin down the corresponding interactions more reliably in the future.

The paper is organized as follows: In Sect. II we describe the employed formalism and in Sect. III we summarize the properties of the $\bar{Y} Y$ potential models used in the calculations. Numerical results for the $e^{+} e^{-} \rightarrow \bar{Y} Y$ reactions are presented in Sect. IV] Specifically, we show the outcome for the cross sections and for the effective form factors, and we provide predictions for the properties of the electromagnetic form factors $G_{M}$ and $G_{E}$. The paper closes with a Summary. 


\section{FORMALISM}

The reaction $e^{+} e^{-} \rightarrow \bar{Y} Y$ is studied with the same formalism as applied by us to $e^{+} e^{-} \rightarrow \bar{p} p$, which was developed and described in detail in Ref. [36]. We summarize it briefly below. We adopt the standard conventions [10] so that the differential cross section for the reactions $e^{+} e^{-} \rightarrow \bar{Y} Y$ is given by

$$
\frac{d \sigma}{d \Omega}=\frac{\alpha^{2} \beta}{4 s} C(s)\left[\left|G_{M}^{Y}(s)\right|^{2}\left(1+\cos ^{2} \theta\right)+\frac{4 M_{Y}^{2}}{s}\left|G_{E}^{Y}(s)\right|^{2} \sin ^{2} \theta\right] .
$$

where $Y$ generically denotes the hyperons $\Lambda, \Sigma$, and $\Xi$, and $\bar{Y}$ stands for the corresponding anti-particles. Here, $\alpha=1 / 137.036$ is the fine-structure constant and $\beta=k_{Y} / k_{e}$ a phase-space factor, where $k_{Y}$ and $k_{e}$ are the center-ofmass three-momenta in the $\bar{Y} Y$ and $e^{+} e^{-}$systems, respectively, related to the total energy via $\sqrt{s}=2 \sqrt{M_{Y}^{2}+k_{p}^{2}}=$ $2 \sqrt{m_{e}^{2}+k_{e}^{2}}$. Further, $m_{e}\left(M_{Y}\right)$ is the electron $(Y)$ mass. The $S$-wave Sommerfeld-Gamow factor $C(s)$ is given by $C=y /\left(1-e^{-y}\right)$ with $y=\pi \alpha M_{Y} / k_{Y}$. Of course, for uncharged hyperons $\left(\Lambda, \Sigma^{0}, \Xi^{0}\right)$ we have $C(s) \equiv 1$. $G_{E}$ and $G_{M}$ are the electric and magnetic form factors of the hyperons in question. In general, we omit the superscript $Y$ from the $G$ 's in the following because it is anyhow clear from the context which hyperon is discussed. The cross section as written in Eq. (1) results from the one-photon exchange approximation and by setting the electron mass $m_{e}$ to zero (in that case $\beta=2 k_{Y} / \sqrt{s}$ ). We will restrict ourselves throughout this work to the one-photon exchange so that the total angular momentum is fixed to $J=1$ and the $e^{+} e^{-}$and $\bar{Y} Y$ systems can be only in the partial waves ${ }^{3} S_{1}$ and ${ }^{3} D_{1}$. We use the standard spectral notation ${ }^{(2 S+1)} L_{J}$, where $S$ is the total spin and $L$ the orbital angular momentum.

The integrated reaction cross section is readily found to be

$$
\sigma_{e^{+} e^{-} \rightarrow \bar{Y} Y}=\frac{4 \pi \alpha^{2} \beta}{3 s} C(s)\left[\left|G_{M}(s)\right|^{2}+\frac{2 M_{Y}^{2}}{s}\left|G_{E}(s)\right|^{2}\right] .
$$

Another quantity used in various analyses is the effective baryon form factor $G_{\text {eff }}$ which is defined by

$$
\left|G_{\mathrm{eff}}(s)\right|=\sqrt{\frac{\sigma_{e^{+} e^{-} \rightarrow \bar{Y} Y}(s)}{\frac{4 \pi \alpha^{2} \beta}{3 s} C(s)\left[1+\frac{2 M_{Y}^{2}}{s}\right]}} .
$$

In order to implement the FSI we perform a partial wave projection of the $e^{+} e^{-} \rightarrow \bar{Y} Y$ amplitudes. The corresponding formalism is documented in various publications in the literature. We follow here the procedure described in detail in the Appendices $\mathrm{B}$ and $\mathrm{C}$ of Ref. [58]. Then we end up with four amplitudes, corresponding to the coupling between the $e^{+} e^{-}$and the $\bar{Y} Y$ systems and the coupled ${ }^{3} S_{1^{-}}{ }^{3} D_{1}$ partial waves. We can write these in the form $F_{L} L^{\prime}$, where $L^{\prime}(L)=0,2$ characterizes the orbital angular momentum in the initial (final) state. The explicit expressions for the reaction $e^{+} e^{-} \rightarrow \bar{Y} Y$ are

$$
\begin{aligned}
& F_{22}^{\mu, \nu}=-\frac{2 \alpha}{9}\left[G_{M}-\frac{2 M_{Y}}{\sqrt{s}} G_{E}\right]\left[1-\frac{2 m_{e}}{\sqrt{s}}\right], \\
& F_{00}^{\mu, \nu}=-\frac{4 \alpha}{9}\left[G_{M}+\frac{M_{Y}}{\sqrt{s}} G_{E}\right]\left[1+\frac{m_{e}}{\sqrt{s}}\right], \\
& F_{02}^{\mu, \nu}=-\frac{2 \sqrt{2} \alpha}{9}\left[G_{M}+\frac{M_{Y}}{\sqrt{s}} G_{E}\right]\left[1-\frac{2 m_{e}}{\sqrt{s}}\right], \\
& F_{20}^{\mu, \nu}=-\frac{2 \sqrt{2} \alpha}{9}\left[G_{M}-\frac{2 M_{Y}}{\sqrt{s}} G_{E}\right]\left[1+\frac{m_{e}}{\sqrt{s}}\right] .
\end{aligned}
$$

The superscripts in Eq. (4) symbolize the channels where $\nu=e^{+} e^{-}$and $\mu=\bar{Y} Y$.

It is obvious from Eq. (4) that the amplitude $F_{L L^{\prime}}^{\mu, \nu}$ can be written as a product of factors. This is simply a consequence of the one-photon exchange which amounts to an $s$-channel pole diagram in the reactions $e^{+} e^{-} \rightarrow \bar{Y} Y$. The factors correspond to the $e^{+} e^{-} \gamma$ and $\bar{Y} Y \gamma$ vertices, respectively, and reflect whether the coupling occurs in an $S$ or $D$ wave. Thus, we can write the amplitude in the form $\left(L, L^{\prime}=0,2\right)$

$$
\begin{aligned}
F_{L L^{\prime}}^{\mu, \nu} & =-\frac{4 \alpha}{9} f_{L}^{\mu} f_{L^{\prime}}^{\nu}, \quad \text { with } \\
f_{0}^{\mu} & =\left(G_{M}+\frac{M_{Y}}{\sqrt{s}} G_{E}\right), f_{2}^{\mu}=\frac{1}{\sqrt{2}}\left(G_{M}-\frac{2 M_{Y}}{\sqrt{s}} G_{E}\right),
\end{aligned}
$$


and similar expressions for $f_{L}^{\nu}$, the vertex functions of the $e^{+} e^{-}$pair. The FSI effects due to the $\bar{Y} Y$ interaction influence only the $\bar{Y} Y$ vertex and that means only $f_{L}^{\mu}$, see Fig. 1 in Ref. [36]. These effects can be calculated rigorously within our formalism [36] after a proper generalization in order to take into account the possible coupling between the various $\bar{Y} Y$ channels. Considering such a coupling, Eq. (8) in the above reference then reads

$$
f_{L^{\prime}}^{\mu^{\prime}}\left(k ; E_{k}\right)=f_{L^{\prime}}^{0\left(\mu^{\prime}\right)}(k)+\sum_{\mu} \sum_{L} \int_{0}^{\infty} \frac{d p p^{2}}{(2 \pi)^{3}} f_{L}^{0(\mu)}(p) \frac{1}{2 E_{k}-2 E_{p}^{\mu}+i 0^{+}} T_{L L^{\prime}}^{\mu, \mu^{\prime}}\left(p, k ; E_{k}\right),
$$

where $\mu, \mu^{\prime}$ stand for any of the $\bar{Y} Y$ channels. For each $\bar{Y} Y$ channel the first term on the right-hand side, the so-called Born term, represents the bare $\bar{Y} Y$ production vertex $f_{L}^{0\left(\mu^{\prime}\right)}$ and the integral provides the dressing of this vertex via rescattering in all the (coupled) $\bar{Y} Y$ channels. The quantity $T_{L L^{\prime}}^{\mu, \mu^{\prime}}\left(p, p^{\prime} ; E_{k}\right)$ is the $\bar{Y} Y$ scattering amplitude in the coupled ${ }^{3} S_{1^{-}}{ }^{3} D_{1}$ partial wave and is the solution of a corresponding Lippmann-Schwinger equation:

$$
T_{L^{\prime \prime} L^{\prime}}^{\mu^{\prime \prime}, \mu^{\prime}}\left(p^{\prime \prime}, p^{\prime} ; E_{k}\right)=V_{L^{\prime \prime} L^{\prime}}^{\mu^{\prime \prime}, \mu^{\prime}}\left(p^{\prime \prime}, p^{\prime}\right)+\sum_{\mu} \sum_{L} \int_{0}^{\infty} \frac{d p p^{2}}{(2 \pi)^{3}} V_{L^{\prime \prime} L}^{\mu^{\prime \prime}, \mu}\left(p^{\prime \prime}, p\right) \frac{1}{2 E_{k}-2 E_{p}^{\mu}+i 0^{+}} T_{L L^{\prime}}^{\mu, \mu^{\prime}}\left(p, p^{\prime} ; E_{k}\right)
$$

see Ref. [55]. In the above equations, $2 E_{k}=2 \sqrt{M_{Y}^{2}+k^{2}}=\sqrt{s}$, where $k$ is the $\bar{Y} Y$ on-shell momentum.

The bare $\bar{Y} Y \gamma$ vertex functions, $f_{L}^{0(\mu)}(L=0,2)$ in Eq. (6) $)$, can be written in terms of bare EMFFs, $G_{E}^{0(\mu)}$ and $G_{M}^{0(\mu)}$, in complete analogy to Eq. (5). In general, $f_{0}^{0(\mu)}$ and $f_{2}^{0(\mu)}$ can depend on the total energy and on the (off-shell) momentum of the $\bar{Y} Y$ system. Furthermore, they are complex because $f_{L}^{0(\mu)}$ (or, equivalently, $G_{E}^{0(\mu)}$ and $G_{M}^{0(\mu)}$ ) involve implicitly contributions from intermediate mesonic states such as $\gamma \rightarrow \pi^{+} \pi^{-} \rightarrow \bar{Y} Y, \gamma \rightarrow K^{+} K^{-} \rightarrow \bar{Y} Y$, etc.

In the present study we assume that the entire energy dependence of the dressed vertex functions $f_{L}^{\mu}$ is generated by the FSI alone and that $f_{0}^{0(\mu)}$ and $f_{2}^{0(\mu)}$ themselves are energy-independent. In particular, we interpret the explicit dependence of $f_{L}^{0(\mu)}$ on $\sqrt{s}$ that is implied by Eq. (5) as a dependence on the momentum of the $\bar{Y} Y$ system. Accordingly, we use

$$
\begin{aligned}
& f_{0}^{0(\mu)}(p)=\left(G_{M}^{0(\mu)}+\frac{M_{Y}}{2 E_{p}} G_{E}^{0(\mu)}\right)=\left(G_{M}^{0(\mu)}+\frac{M_{Y}}{2 \sqrt{M_{Y}^{2}+p^{2}}} G_{E}^{0(\mu)}\right), \\
& f_{2}^{0(\mu)}(p)=\frac{1}{\sqrt{2}}\left(G_{M}^{0(\mu)}-\frac{M_{Y}}{E_{p}} G_{E}^{0(\mu)}\right)=\frac{1}{\sqrt{2}}\left(G_{M}^{0(\mu)}-\frac{M_{Y}}{\sqrt{M_{Y}^{2}+p^{2}}} G_{E}^{0(\mu)}\right),
\end{aligned}
$$

for the bare vertex functions, where $p$ is the center-of-mass momentum in the $\bar{Y} Y$ system. Note that the replacement $\sqrt{s} \rightarrow 2 E_{p}$ is required in order to guarantee the correct threshold behavior of the $D$-wave vertex function $f_{2}^{0}(p)$ which has to behave like $\propto p^{2}$. Indeed, Eqs. (5) or (8) reveal that the condition $G_{E}^{0}=G_{M}^{0}$ and/or $G_{E}=G_{M}$ in the timelike region at the $\bar{Y} Y$ thresholds [10] is equivalent to implementing the proper threshold behavior of the $D$-wave amplitude.

We assume that $G_{E}^{0}$ and $G_{M}^{0}$ are constants which automatically implies that we have to set $G_{E}^{0}=G_{M}^{0} \cdot G_{E}^{0}\left(G_{M}^{0}\right)$ were taken to be real in our studies of the reactions $e^{+} e^{-} \rightarrow \bar{p} p$ and $e^{+} e^{-} \rightarrow \bar{\Lambda} \Lambda$ because any overall phase drops out in the evaluation of observables. In that case there is only a single free parameter in the calculation, which is basically a normalization constant. In a coupled-channel approach, cf. Eq. (6), there is a $G_{E}^{0}\left(G_{M}^{0}\right)$ for each channel and only one of them can be chosen to be real. However, in the present investigations of the hyperon form factores we will consider such a coupling only between the $\bar{\Sigma} \Sigma$ channels, i.e. for $\bar{\Sigma}^{-} \Sigma^{+}, \bar{\Sigma}^{0} \Sigma^{0}, \bar{\Sigma}^{+} \Sigma^{-}$. In this case the reaction thresholds are very close together and the channels strongly influence each other. Moreover, experimental information is available for all three channels. Then there are three constants that need to be fixed from the data, where one of them can be chosen real. The bare form factors for the other reactions $\left(\bar{\Lambda} \Lambda, \bar{\Sigma}^{0} \Lambda, \bar{\Xi} \Xi\right)$ are fixed independently and are all chosen to be real.

The bare vertex functions $f_{0}^{0}$ and $f_{2}^{0}$ are calculated from Eq. (8) and inserted into Eq. (6). Due to the FSI the resulting dressed vertex functions $f_{0}$ and $f_{2}$ are always energy-dependent and complex. Inverting Eq. (5) we can obtain $G_{E}$ and $G_{M}$ and then evaluate any $e^{+} e^{-} \rightarrow \bar{Y} Y$ observable based on the formulae provided at the beginning of this section. $G_{E}$ and $G_{M}$ are also always complex and, in general, $G_{E} \neq G_{M}$, where the difference is likewise solely due to the FSI.

Note that the formulae given above apply for $e^{+} e^{-} \rightarrow \bar{\Sigma}^{0} \Lambda$ as well, once the $Y$ mass is replaced by $\left(M_{\Sigma^{0}}+M_{\Lambda}\right) / 2$ and, accordingly, $E_{p}$ by $\left(\sqrt{M_{\Sigma^{0}}^{2}+p^{2}}+\sqrt{M_{\Lambda}^{2}+p^{2}}\right) / 2$. 


\section{THE $\bar{Y} Y$ INTERACTION}

In the present work we utilize a $\bar{Y} Y$ interaction that has been established in studies of antihyperon-hyperon production in $\bar{p} p$ collisions [52, 54 56]. In this model of the Jülich group the hyperon-production reaction is considered within a coupled-channel approach. This allows one to take into account rigorously the effects of the initial $(\bar{p} p)$ and final $(\bar{Y} Y)$ state interactions. The interaction in the various $\bar{Y} Y$ channels and the transition between them is described by meson exchanges whose parameters are taken over from studies of the $N N$ [57] and $Y N$ interactions [58] on the basis of a $G$-parity transformation. The strangeness production process $\bar{p} p \rightarrow \bar{Y} Y$ is given by the exchange of strange mesons [52, 54]. Annihilaton in the $\bar{Y} Y$ channels is taken into account by phenomenological optical potentials. These potentials involve free parameters, which have been fixed by a fit to the $\bar{p} p \rightarrow \bar{Y} Y$ observables. We want to emphasize that the experimental constraints in the various $\bar{Y} Y$ channels are of rather different quality. The reaction $\bar{p} p \rightarrow \bar{\Lambda} \Lambda$ has been extensively investigated in the PS185 experiment and data are available for total and differential cross sections as well as for spin-dependent observables [44 47], down to energies very close to the reaction threshold, see e.g. the review in Ref. [48]. For the reaction $\bar{p} p \rightarrow \bar{\Sigma}^{0} \Lambda+$ c.c. the situation is already less satisfactory and for $\bar{p} p \rightarrow \Sigma^{-} \Sigma^{+}$ and $\bar{p} p \rightarrow \bar{\Sigma}^{+} \Sigma^{-}$basically only a single measurement exists. In case of $\bar{p} p \rightarrow \bar{\Xi}$ only upper bounds of the reaction cross section are available. The quality of the description of the PS185 data by the Jülich model is documented in Refs. [52, 54, 55].

Since the reaction $e^{+} e^{-} \rightarrow \bar{\Sigma} \Sigma$ is discussed in greater detail in the present study we display in Fig. 1 results of the employed $\bar{Y} Y$ model 55] for $\bar{p} p \rightarrow \bar{\Sigma} \Sigma$. Another reason is that at the time when the model was published only a (preliminary) data point for the $\bar{\Sigma}^{-} \Sigma^{+}$channel was available. The results for $\bar{p} p \rightarrow \bar{\Sigma}^{+} \Sigma^{-}$were, therefore, genuine predictions. It is re-assuring to see that the model predictions are well in line with the final data, in particular, in view of the fact that $\bar{p} p \rightarrow \bar{\Sigma}^{+} \Sigma^{-}$requires double-charge exchange, i.e. involves at least a two-step process.

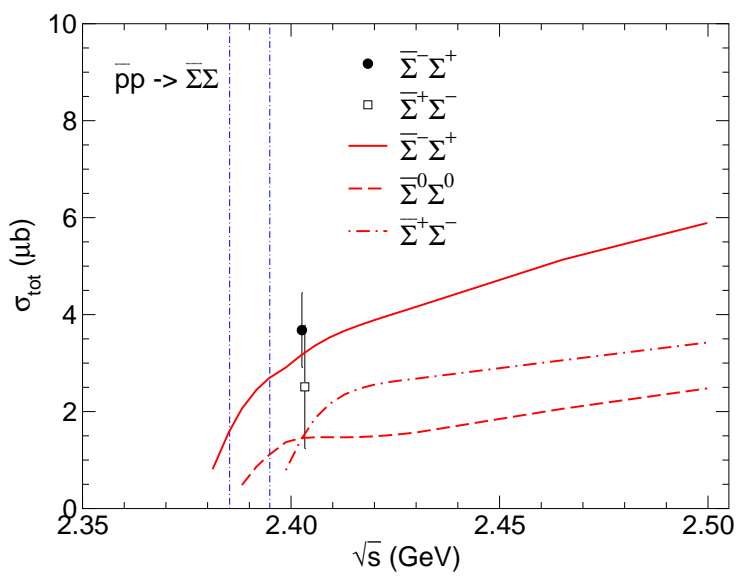

FIG. 1. Results for $\bar{p} p \rightarrow \bar{\Sigma} \Sigma$ based on the $\bar{Y} Y$ model presented in Ref. [55]. Data are taken from Refs. 49 51]. The horizontal dash-dotted lines indicate the $\bar{\Sigma}^{0} \Sigma^{0}$ and $\bar{\Sigma}^{+} \Sigma^{-}$thresholds.

Partial wave cross sections at the excess energy of $\epsilon=24 \mathrm{MeV}$ are listed in Table 3 of Ref. 55. One can see that the by-far largest contribution comes from the ${ }^{3} P_{2}$ partial wave, despite of the low energy. The contribution of the ${ }^{3} S_{1}$ amounts to only $30 \%\left(\bar{\Sigma}^{-} \Sigma^{+}\right)$and $10 \%\left(\bar{\Sigma}^{+} \Sigma^{-}\right)$of the $\bar{p} p \rightarrow \bar{\Sigma} \Sigma$ transition cross section. In the meson-exchange potential the cross section is driven by the strong (and coherent) tensor forces due to $K$ and $K^{*}$ exchange which leads to the characteristic dominance of $L \rightarrow L-2$ transitions [55]. This will be certainly different for $e^{+} e^{-} \rightarrow \bar{\Sigma} \Sigma$ where the prime mechanism is $s$-channel one-photon exchange.

Predictions of the model for the total $\bar{\Sigma} \Sigma$ cross sections and for $\bar{\Lambda} \Lambda \rightarrow \bar{\Sigma} \Sigma$ and $\bar{\Lambda} \Sigma \rightarrow \bar{\Sigma} \Sigma$ transition cross sections can be found in Figs. 23 and 24 of Ref. [55]. Note that the transition cross sections are shown in $\mu b$ and suggest that the coupling to $\bar{\Sigma} \Sigma$ is relatively week. In view of that, we do not take into account those channels explicitly in the evaluation of the $\bar{\Sigma} \Sigma$ form factors via Eq. (6). Rather we assume that their effect can be absorbed into the "bare" form factors, which anyway involve free parameters that need to be determined by a fit to the BESIII data.

The limited amount of data for $\bar{p} p \rightarrow \bar{\Sigma} \Sigma$ did not require a refined adjustment of the $\bar{\Sigma} \Sigma$ interaction. Specifically, the annihilation part is described only by a central potential. Unlike the situation for $\bar{\Lambda} \Lambda$ and $\bar{\Sigma}^{0} \Lambda$ no LS or tensor component was used because the corresponding strength and range parameters could not be fixed form the single 

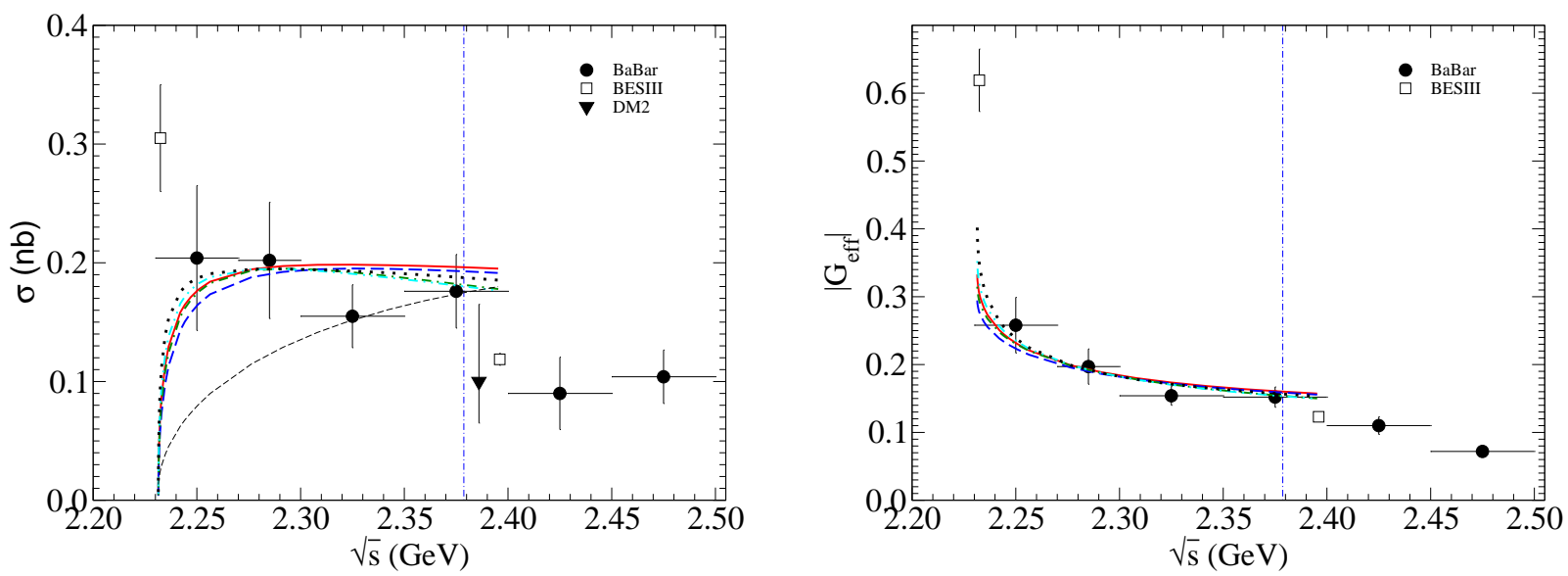

FIG. 2. $e^{+} e^{-} \rightarrow \bar{\Lambda} \Lambda$ : Cross section (left) and effective form factor $\left|G_{\text {eff }}\right|$ (right) as a function of the total cms energy. The solid, dashed, and dash-dotted lines correspond to the $\bar{\Lambda} \Lambda$ models I, II, and III from Ref. [52], the dash-double-dotted and dotted lines to the models K and Q described in Ref. [54]. Data are from the Refs. [11] (DM2), 12] (BaBar), and [19, 20] (BESIII). The phase space is shown by the thin (black) dashed line. The horizontal dash-dotted line indicates the isospin-averaged $\bar{\Sigma} \Sigma$ threshold.

data point available at that time 55 .
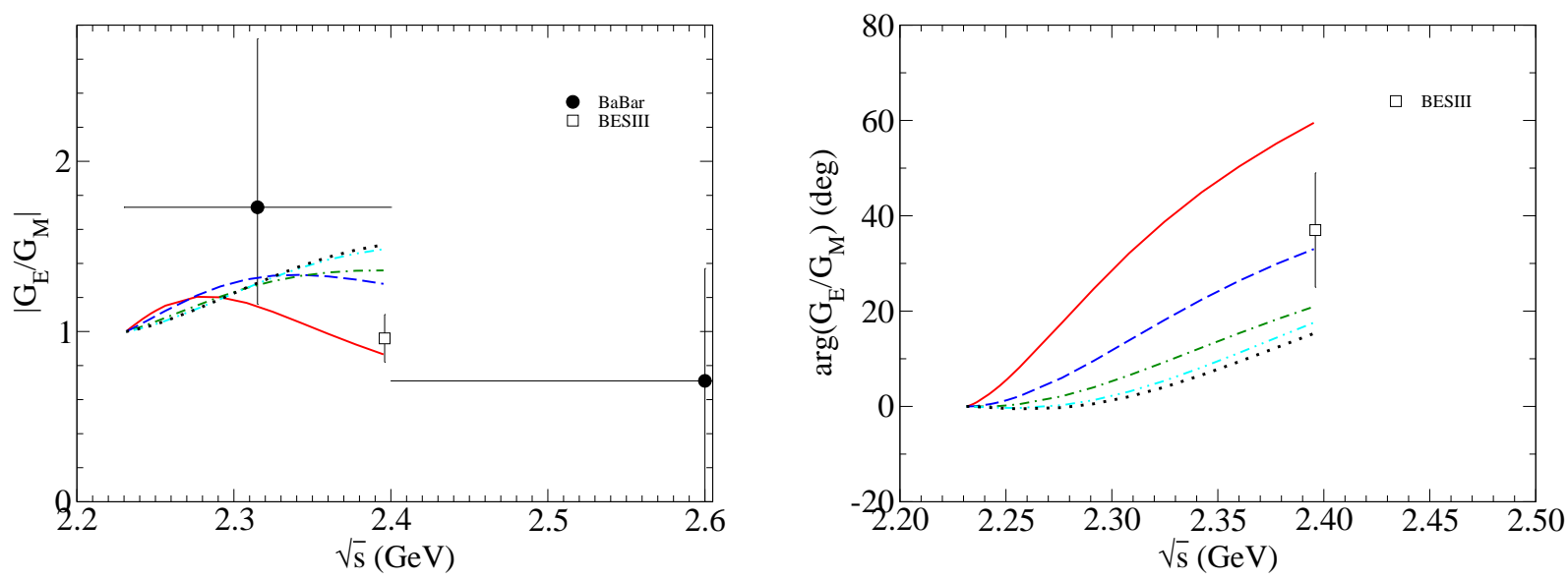

FIG. 3. $e^{+} e^{-} \rightarrow \bar{\Lambda} \Lambda$ : The ratio $\left|G_{E} / G_{M}\right|$ (left) and phase $\phi=\arg \left(\mathrm{G}_{\mathrm{E}} / \mathrm{G}_{\mathrm{M}}\right)$ (right) as a function of the total cms energy. Same description of curves as in Fig. 2] Data are from Refs. [12] (BaBar) and 20] (BESIII).

\section{RESULTS FOR $\bar{Y} Y$}

$$
\text { A. } e^{+} e^{-} \rightarrow \bar{\Lambda} \Lambda
$$

Our results for $e^{+} e^{-} \rightarrow \bar{\Lambda} \Lambda$ have been discussed in detail in Ref. [24]. We reproduce some of them here because at the time when our work was published only preliminary results from the BESIII Collaboration were available. In the meantime final results have been presented [19]. As can be seen in Fig. 2 the initially reported rather large cross section extremely close to the threshold has been confirmed. Its value is not in line with the trend suggested by the BaBar data and, also, it cannot be reproduced by our $\bar{\Lambda} \Lambda$ FSI. A plausible and convincing explanation/interpretation is still 
missing 25 28]. For a more thorough discussion of this issue see Ref. 24]. Interestingly, the BESIII Collaboration has accomplished a complete measurement of the $\Lambda$ EMFFs at $2.396 \mathrm{MeV}$, which produced experimental values for the ratio $\left|G_{E} / G_{M}\right|$ and for the phase between $G_{E}$ and $G_{M}$, i.e. $\phi=\arg \left(\mathrm{G}_{\mathrm{E}} / \mathrm{G}_{\mathrm{M}}\right)$ [20]. Those data are confronted with our predictions in Fig. 3. Evidently, among the various models considered in our study 24], the results based on model I from Ref. [52] are closest to the measurement. Note that $\left|G_{E} / G_{M}\right|$ is shown for an expanded energy range so that one can see better the trend for this observable as suggested by the BaBar data point at $2.6 \mathrm{GeV}$.
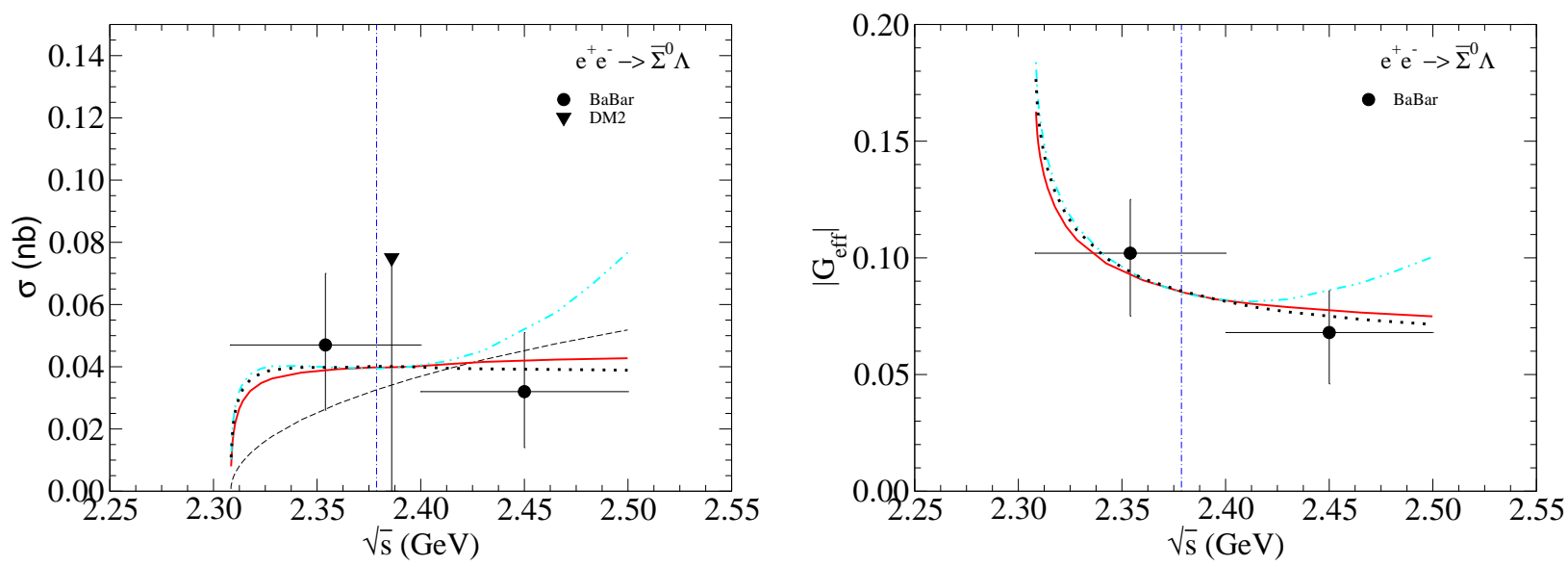

FIG. 4. $e^{+} e^{-} \rightarrow \bar{\Sigma}^{0} \Lambda$ : Cross section (left) and effective form factor $\left|G_{\text {eff }}\right|$ (right). The solid, dash-double-dotted and dotted lines correspond to the models I, K and Q described in Ref. [55]. Data points are taken from the DM2 [1] and BaBar [12] Collaborations. The phase space is shown by the thin (black) dashed line. The horizontal dash-dotted line indicates the $\bar{\Sigma} \Sigma$ threshold.
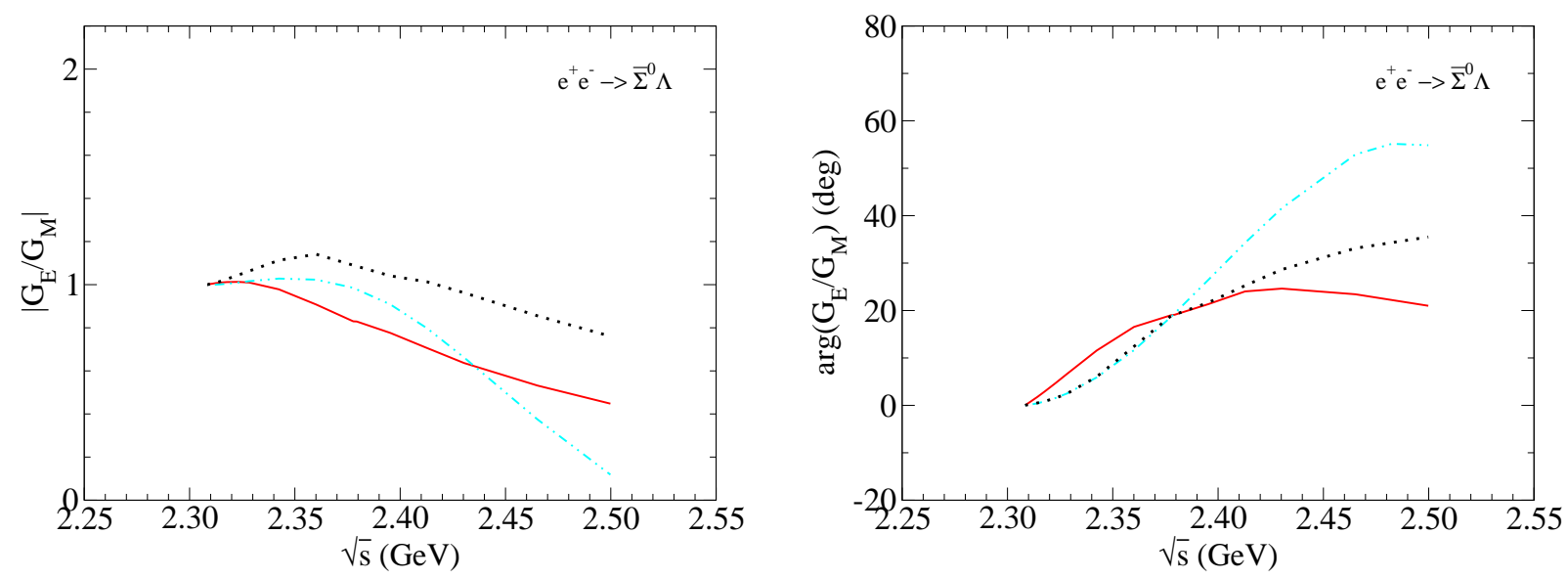

FIG. 5. $e^{+} e^{-} \rightarrow \bar{\Sigma}^{0} \Lambda$ : Ratio $\left|G_{E} / G_{M}\right|$ (left) and phase $\phi=\arg \left(\mathrm{G}_{\mathrm{E}} / \mathrm{G}_{\mathrm{M}}\right)$ (right). Same description of curves as in Fig. 4

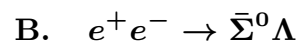

Results for $e^{+} e^{-} \rightarrow \bar{\Sigma}^{0} \Lambda$ are presented in Figs. 4 and 5. For that reaction only cross sections are avaible [12]. The data point from DM2 [11] is an upper limit. We fixed the normalization (i.e. the bare form factor) so that the cross sections are $0.04 \mathrm{nb}$ at $2.4 \mathrm{GeV}$ for all three $\bar{\Sigma}^{0} \Lambda$ models presented in [55]. Comparing Figs. 4 and 2 one can see that the $\bar{\Sigma}^{0} \Lambda$ cross section is about a factor 5 smaller than that for $\bar{\Lambda} \Lambda$. However, like the latter one it remains practically 


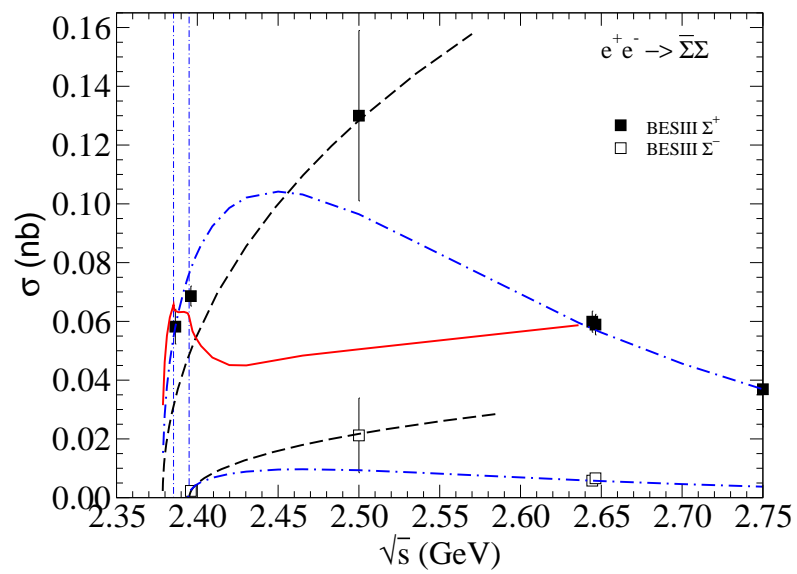

FIG. 6. $e^{+} e^{-} \rightarrow \bar{\Sigma} \Sigma$ : Illustrative curves/results for the $\bar{\Sigma}^{-} \Sigma^{+}$and $\bar{\Sigma}^{+} \Sigma^{-}$cross sections. The dashed lines represent the phase space behavior. The solid line is the result of an exploratory calculation that uses only the $\bar{\Sigma}^{-} \Sigma^{+} \rightarrow \bar{\Sigma}^{-} \Sigma^{+}$amplitude for the FSI. The dash-dotted line represents the pQCD motivated parameterization Eq. (3) in [21]. Data are taken from Ref. [21]. The horizontal lines indicate the $\bar{\Sigma}^{0} \Sigma^{0}$ and $\bar{\Sigma}^{+} \Sigma^{-}$thresholds.

constant over a fairly large energy region above the threshold and does not follow the phase space behavior. This conclusion can be savely drawn despite of the modest energy resolution. The results based on two of the $\bar{Y} Y$ models considered in Ref. 55] agree nicely with that behavior over the whole considered energy region. One of the models suggest a rise in the cross section from around $2.4 \mathrm{GeV}$ onwards which is clearly ruled out by the experiment. As can be seen in Fig. [5] also for the ratio $\left|G_{E} / G_{M}\right|$ and the phase $\phi=\arg \left(\mathrm{G}_{\mathrm{E}} / \mathrm{G}_{\mathrm{M}}\right)$ a qualitative similar behavior to that for the $\bar{\Lambda} \Lambda$ channel is predicted.

\section{C. $e^{+} e^{-} \rightarrow \bar{\Sigma} \Sigma$}

Let us begin the discussion on the $\bar{\Sigma} \Sigma$ channel with some general considerations. Alternatively to [21], in Fig. 6] we present the BESIII results up to $2.75 \mathrm{GeV}$ on a linear scale. Data for $\bar{\Sigma}^{-} \Sigma^{+}$(filled squares) and $\bar{\Sigma}^{+} \Sigma^{-}$(opaque squares) are included together with their statistical uncertainties. The systematic uncertainties quoted in the paper are usually of the same magnitude [21]. The pure phase space behavior corresponds to the dashed lines. The results based on the pQCD motivated fit via Eq. (3) in Ref. [21] are indicated by the dash-dotted lines. The result of an exploratory calculation based on the $\bar{Y} Y$ model from Ref. [55], where only the $\bar{\Sigma}^{-} \Sigma^{+}$amplitude is taken into account in Eq. (6), is shown by the solid line. The horizontal dashed lines indicate the $\bar{\Sigma}^{0} \Sigma^{0}$ and $\bar{\Sigma}^{+} \Sigma^{-}$thresholds.

Evidently, the first two data points for $\bar{\Sigma}^{-} \Sigma^{+}$are close to the thresholds of the $\bar{\Sigma}^{0} \Sigma^{0}$ and $\bar{\Sigma}^{+} \Sigma^{-}$channels, respectively. Furthermore, the first three data points are roughly in line with the phase-space behavior, if one takes into account the large uncertainty of the measurement at $\sqrt{s}=2.5 \mathrm{GeV}$. At first sight this suggest that, unlike what was observed for $\bar{\Lambda} \Lambda$ and $\bar{\Sigma}^{0} \Lambda$, FSI effects could be very small in case of $\bar{\Sigma} \Sigma$. However, the next two data points, around $2.65 \mathrm{GeV}$, suggest a quite different energy dependence. These cross sections are of comparable magnitude to the ones around $2.39 \mathrm{MeV}$ and would be in line with a basically flat behavior similar to what has been seen for $\bar{\Lambda} \Lambda$ and $\bar{\Sigma}^{0} \Lambda$. We include here the results based on the pQCD inspired function considered in the BESIII paper, cf. Eq. (3) in Ref. [21]. It allows one to reproduce the energy dependence of the cross section quite well over the whole energy range, except for the data point at $2.5 \mathrm{GeV}$. The situation for the $\bar{\Sigma}^{+} \Sigma^{-}$channel is similar. Also here the cross section at $2.5 \mathrm{GeV}$ is not described.

We take the above observations as a strong indication against using the cross sections at $2.5 \mathrm{GeV}$ for fixing the parameters of our bare form factors (8). Instead we utilize those around $2.65 \mathrm{GeV}$. We want to emphasize that this choice is certainly debatable. The two points in question are already more than $200 \mathrm{MeV}$ away from the $\bar{\Sigma} \Sigma$ thresholds and, therefore, our assumption that the bare form factors are constant is most likely not valid over such a large energy range. One should keep this caveat in mind when assessing the results that will be presented below.

In the actual fitting process we also ignored the measured ratio $\left|G_{E} / G_{M}\right|$ at $2.396 \mathrm{GeV}$. Here the BESIII Collaboration found a value of $1.83 \pm 0.26$ [21] which is extremely large given that this energy is just about $17 \mathrm{MeV}$ away from the $\bar{\Sigma}^{-} \Sigma^{+}$threshold. Since $G_{E}=G_{M}$ at the threshold, as mentioned above, one would need an excessively large 

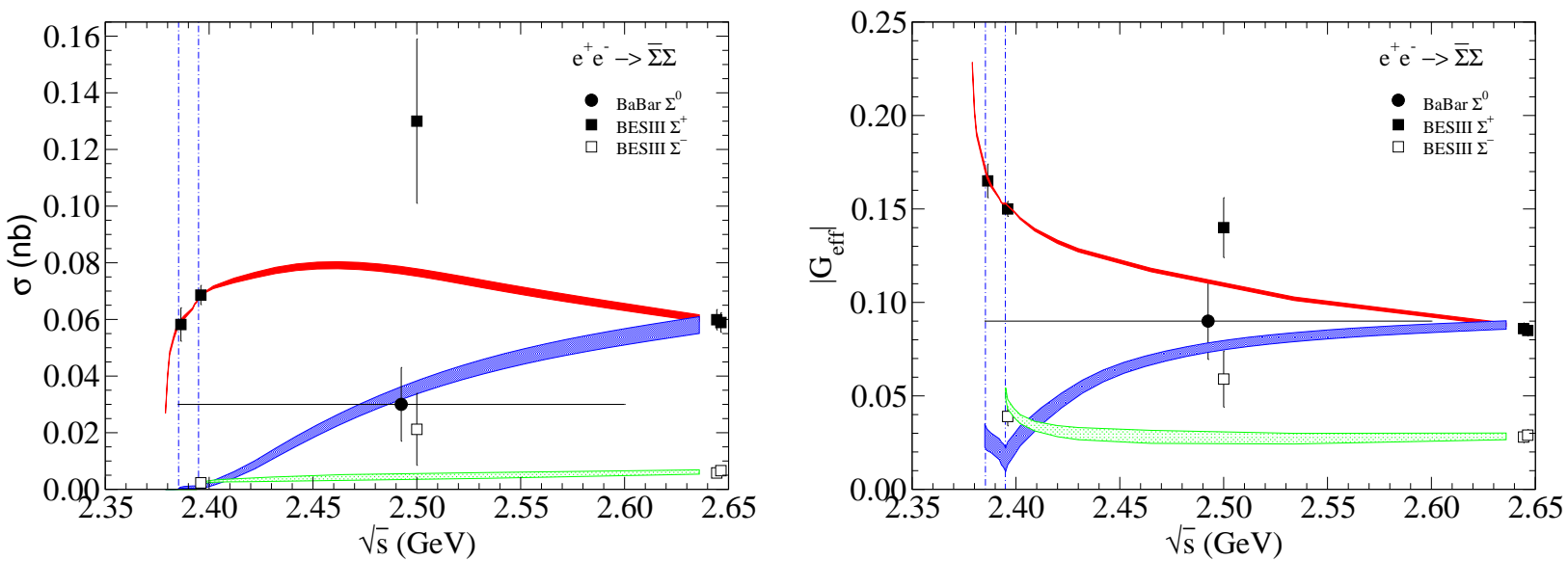

FIG. 7. $e^{+} e^{-} \rightarrow \bar{\Sigma} \Sigma$ : Cross section (left) and effective form factor $\left|G_{\text {eff }}\right|$ (right). Results are shown based on the $\bar{\Sigma} \Sigma$ interaction from Ref. [55], where the dark (red) bands correspond to $\bar{\Sigma}^{-} \Sigma^{+}$, medium (blue) bands to $\bar{\Sigma}^{0} \Sigma^{0}$, and light (green) bands to $\bar{\Sigma}^{+} \Sigma^{-}$. For the meaning of the bands see text. Data for the $\bar{\Sigma}^{-} \Sigma^{+}$and $\bar{\Sigma}^{+} \Sigma^{-}$channels are from BESIII [21], those for $\bar{\Sigma}^{0} \Sigma^{0}$ from BaBar [12]. The horizontal dash-dotted lines indicate the $\bar{\Sigma}^{0} \Sigma^{0}$ and $\bar{\Sigma}^{+} \Sigma^{-}$thresholds.
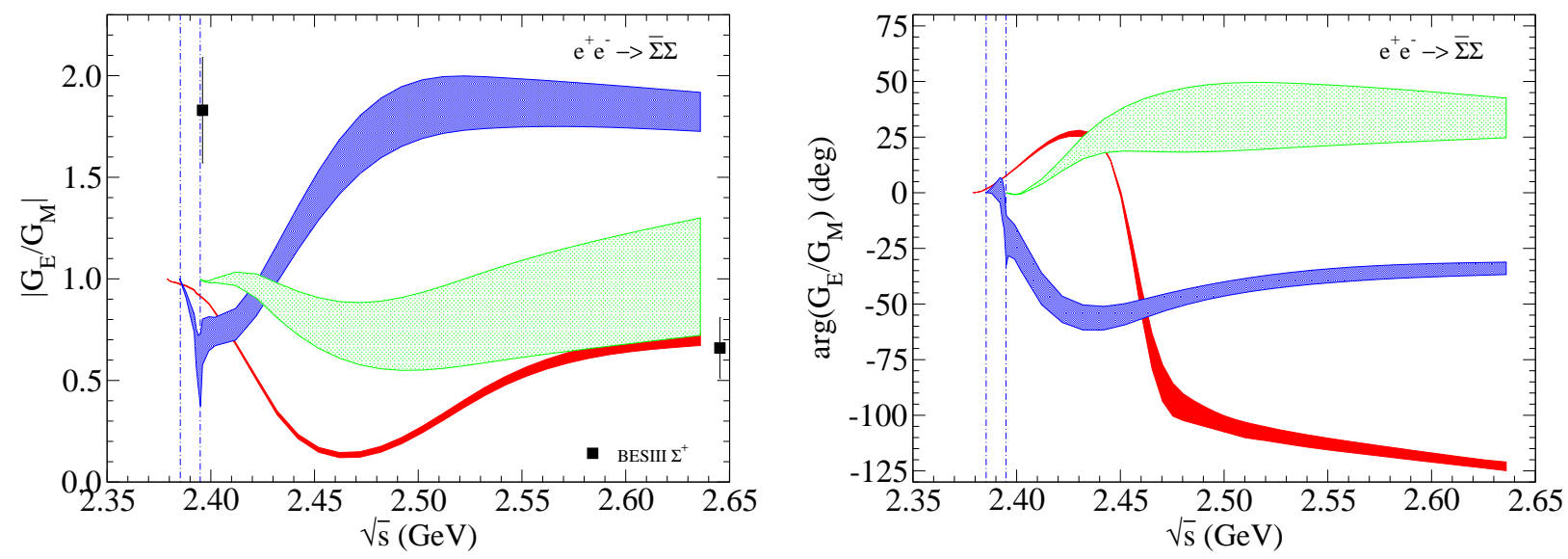

FIG. 8. $e^{+} e^{-} \rightarrow \bar{\Sigma} \Sigma$ : Ratio $\left|G_{E} / G_{M}\right|$ (left) and phase $\phi=\arg \left(\mathrm{G}_{\mathrm{E}} / \mathrm{G}_{\mathrm{M}}\right)$ (right). Same description of curves as in Fig. 7 Data for $\bar{\Sigma}^{-} \Sigma^{+}$are from the BESIII Collaboraton [21].

$D$-wave component in order to achieve such a value, which cannot be generated within the conventional dynamics of the employed $\bar{Y} Y$ model. Exotic contributions like near-threshold $D$-wave resonances might provide such drastic effects, but we do not want to be too speculative at this stage.

Before proceeding to the actual fit it is worthwhile to look at the exploratory calculation with just the $\bar{\Sigma}^{-} \Sigma^{+}$ included (solid line in Fig. 6). The resulting cross section is normalized to the data points around $2.645 \mathrm{GeV}$. Obviously, then also the predictions around $2.39 \mathrm{GeV}$ are fairly well in line with the experiment. However, more interesting is the energy dependence exhibited in that region. One can clearly see threshold effects at the openings of the $\bar{\Sigma}^{0} \Sigma^{0}$ as well as of the $\bar{\Sigma}^{+} \Sigma^{-}$channel. This is a clear signal that the coupling between the $\bar{\Sigma} \Sigma$ channels plays an important role for the $e^{+} e^{-}$cross sections, at least within the employed $\bar{Y} Y$ model, and we ought to include it in the actual calculation.

Results of our fit are shown in Figs. 7 and 8 . In this fit the cross sections at 2.3864, 2.3960, 2.6444, and 2.6464 GeV are included and in addition the ratio $\left|G_{E} / G_{M}\right|$ at the latter two energies. The free parameters are the values of $G_{M}^{0}$ for $\bar{\Sigma}^{-} \Sigma^{+}, \bar{\Sigma}^{0} \Sigma^{0}$, and $\bar{\Sigma}^{+} \Sigma^{-}$that enter the corresponding vertex functions $f_{0}^{0}$ and $f_{2}^{0}$, see Eqs. (8) . $G_{M}^{0\left(\Sigma^{+}\right)}$is chosen 
to be real, since the overall phase drops out in the calculation of the observables anyway, the other two are complex. Note that $G_{M}^{0(Y)} \equiv G_{E}^{0(Y)}$ so that there are no further parameters. The results are normalized to the $\bar{\Sigma}^{-} \Sigma^{+}$cross section at $2.3960 \mathrm{GeV}$. However, we have checked that choosing a different energy for normalization leaves the results practically unchanged.

As a consequence of the lack of data, only a single $\bar{\Sigma} \Sigma$ interaction has been presented in Ref. [55]. It turned out that a comparable description of the data points could be obtained for a range of parameters for the three bare form factors. Therefore, we present the achieved results as bands in Figs. 7 and 8 which visualize this uncertainty. A typical example for the relative weights of the bare form factors as they come out of the fitting procedure is $\bar{\Sigma}^{-} \Sigma^{+} / \bar{\Sigma}^{0} \Sigma^{0} / \bar{\Sigma}^{+} \Sigma^{-}=(1.00,0.00) /(0.55,0.30) /(0.00,0.60)$. Obviously the reproduction of the measured cross section ratio, $\sigma_{\bar{\Sigma}^{-} \Sigma^{+}} / \sigma_{\bar{\Sigma}^{+} \Sigma^{-}} \approx 10$, requires that $G_{M}^{0\left(\Sigma^{+}\right)}$and $G_{M}^{0\left(\Sigma^{-}\right)}$are basically orthogonal.

As one can see from Fig. 7, the data points included in the fit are quite well described. The $\bar{\Sigma}^{-} \Sigma^{+}$and $\bar{\Sigma}^{+} \Sigma^{-}$ cross sections exhibit a weak energy dependence that is more moderate than the one of the "pQCD" parameterization [21]. This is not least due to the fact that the two near-threshold cross sections at 2.3864 and $2.3960 \mathrm{GeV}$ are quite well described, which is not the case for that parameterization, see the dash-dotted line in Fig. 6. Interestingly, also the data point for $\bar{\Sigma}^{0} \Sigma^{0}$ from the BaBar Collaboration [12] is described, though it was not included in the fit. Admittedly, the energy resolution is much more modest than that of the BESIII data. Overall, however, the predicted energy dependence for $\bar{\Sigma}^{0} \Sigma^{0}$ differs from the ones for the other two channel. The difference in the predicted energy dependence for $\bar{\Sigma}^{0} \Sigma^{0}$ can be seen likewise in the results for the effective form factors $\left|G_{\text {eff }}\right|$ shown in the lower panel of Fig. 7. That figure reveals also another special feature of our $\bar{\Sigma}^{0} \Sigma^{0}$ result, namely a pronounced structure at the $\bar{\Sigma}^{+} \Sigma^{-}$threshold. In fact, there is also a structure in the $\bar{\Sigma}^{-} \Sigma^{+}$results at the $\bar{\Sigma}^{0} \Sigma^{0}$ and $\Sigma^{+} \Sigma^{-}$thresholds. But those are rather moderate and, therefore, can be barely seen in the figure.

In Fig. 8 predictions for the ratio $\left|G_{E} / G_{M}\right|$ and the phase $\phi=\arg \left(G_{E} / G_{M}\right)$ are presented. Obviously these observables reflect much more strongly the complicated dynamics in the coupled $\bar{\Sigma} \Sigma$ channels than the cross sections discussed above. It is also worthwhile to recall that the same quantities show a fairly smooth energy dependence in the $\bar{\Lambda} \Lambda$ and $\bar{\Sigma}^{0} \Lambda$ cases. Anyway, one can see that the ratio $\left|G_{E} / G_{M}\right|$ for $\bar{\Sigma}^{-} \Sigma^{+}$drops considerably within the first $50 \mathrm{MeV}$ from the threshold and then increases again to reach the (fitted) BESIII value of around 0.65 at $2.64 \mathrm{GeV}$. Even more spectacular is the behavior of the ratio in case of $\Sigma^{0} \Sigma^{0}$ which shows an extremely strong variation with energy within a very small energy region. In particular, there is a pronounced cusp effect at the opening of the $\bar{\Sigma}^{+} \Sigma^{-}$. Clearly in view of that observation one can speculate that the large ratio measured by the BESIII Collaboration for $\bar{\Sigma}^{-} \Sigma^{+}$near the $\bar{\Sigma}^{+} \Sigma^{-}$threshold might be also a signal for such a cusp effect. The $\bar{Y} Y$ interaction by the Jülich group does not give rise to a more pronounced effect in the $\bar{\Sigma}^{-} \Sigma^{+}$amplitude. However, as already said above, the rather limited information on the $\bar{p} p \rightarrow \bar{\Sigma} \Sigma$ reaction certainly did not allow to constrain the interaction in the $\bar{\Sigma} \Sigma$ channel in that model in a reliable way. It goes without saying that more extended measurements in the region of the $\bar{\Sigma} \Sigma$ thresholds would definitely provide further insight into the $\bar{Y} Y$ dynamics. In particular, it would be useful to explore the energy dependence of observables like the form factor ratio in detail.

We have explored also alternative fit strategies. First we omitted the ratio $\left|G_{E} / G_{M}\right|$ for $\bar{\Sigma}^{-} \Sigma^{+}$at $2.64 \mathrm{GeV}$. Then there is a stronger variation in the predictions for $\left|G_{E} / G_{M}\right|$, e.g. it is in the range of 0.7 and 1.2 at that energy, while the $\chi^{2}$ values for the cross sections remain basically the same. In addition, also the ratios for $\bar{\Sigma}^{0} \Sigma^{0}$ and $\Sigma^{+} \Sigma^{-}$show larger variations. Including the cross sections at $2.5 \mathrm{GeV}$ into the fit does not change the overall results much because of their comparatively large experimental uncertainties. Specifically, the energy dependence of the cross sections remain practically unchanged and only the overall $\chi^{2}$ increases noticeably.

\section{D. $e^{+} e^{-} \rightarrow \bar{\Xi} \Xi$}

Finally, and as a curiosity, we present here also results for the reaction $e^{+} e^{-} \rightarrow \Xi \Xi$, see Figs. 9 and 10 . Those are based on a model used in an exploratory calculation for $\bar{p} p \rightarrow \Xi \Xi[56$. However, since only upper limits for the cross sections are availabe, it is clear that the $\Xi \Xi$ interaction established in that work is basically the result of an educated guess and not really constrained by empirical information. With only experimental information on $e^{+} e^{-} \rightarrow \bar{\Xi}^{+} \Xi^{-}$ the relative weight of the $\bar{\Xi}^{+} \Xi^{-}$and $\bar{\Xi}^{0} \Xi^{0}$ channels cannot be fixed and, therefore, for simplicity we show the results in the isospin channels. In order to guide the eye we normalized the curves to the data point at $2.9 \mathrm{GeV}$. In any case, as one can see in Fig. 9, the predictions for the cross sections and for $\left|G_{\text {eff }}\right|$ are fairly similar for the two isospins. There is more sensitivity to the details in the ratio $\left|G_{E} / G_{M}\right|$ and phase $\phi=\arg \left(\mathrm{G}_{\mathrm{E}} / \mathrm{G}_{\mathrm{M}}\right)$, see Figs. 10. A comparison with the cross sections from BESIII suggests that the FSI effects generated from the employed $\bar{\Xi} \Xi$ model are, may be, somewhat too strong. Obviously additional data points, say in the region 2.65-2.75 GeV, would be very helpful for drawing reliable conclusions. 

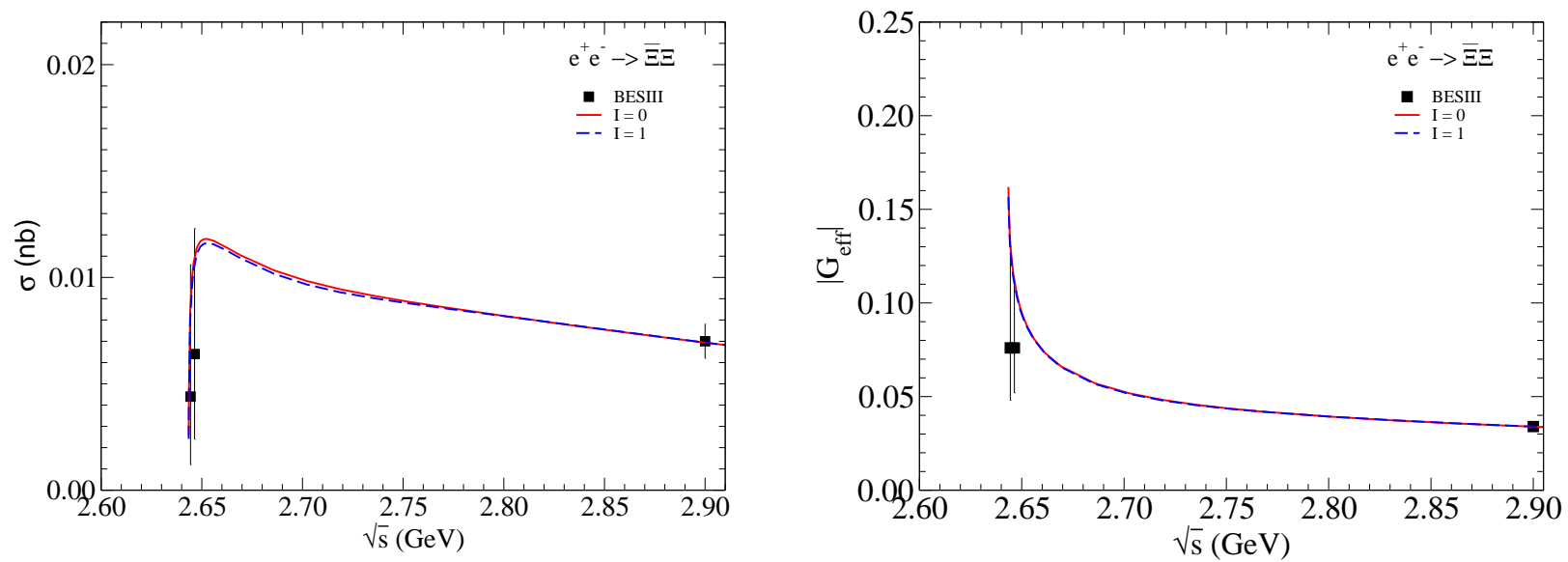

FIG. 9. $e^{+} e^{-} \rightarrow \Xi \Xi$ : Cross section (left) and effective form factor $\left|G_{\text {eff }}\right|$ (right). Results for isospin $I=0$ (solid line) and $I=1$ (dashed line) are shown based on the $\bar{\Xi} \Xi$ interaction from Ref. [56]. Data for the $\bar{\Xi}^{+} \Xi^{-}$channel are from the BESIII Collaboration 23].
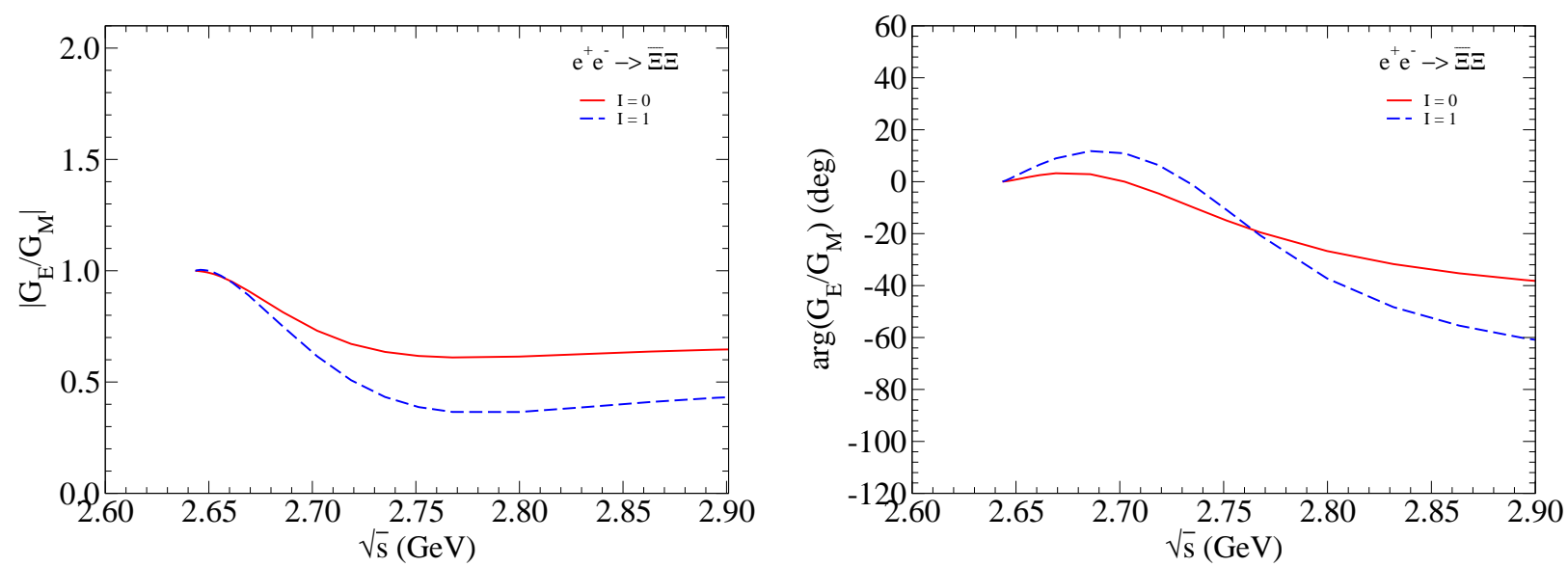

FIG. 10. $e^{+} e^{-} \rightarrow \Xi \Xi$ : Ratio $\left|G_{E} / G_{M}\right|$ (left) and phase $\phi=\arg \left(\mathrm{G}_{\mathrm{E}} / \mathrm{G}_{\mathrm{M}}\right)$ (right). Same description of curves as in Fig. 9 .

\section{SUMMARY}

In the present paper we studied the electromagnetic form factors of hyperons in the timelike region, accessible in the reaction $e^{+} e^{-} \rightarrow \bar{Y} Y$. We focussed on energies near the reaction thresholds and put specific emphasis on the role played by the interaction in the final $\bar{Y} Y$ state. The calculation is based on the one-photon approximation for the elementary reaction mechanism, but takes into account rigorously the effects of the interactions in the $\bar{Y} Y$ systems, in close analogy to our work on $e^{+} e^{-} \rightarrow \bar{p} p[36$. For the $\bar{Y} Y$ interaction we utilized a variety of potential models 52, 54 56 that were established in the analysis of data on the reaction $\bar{p} p \rightarrow \bar{Y} Y$ provided by the PS185 experiment at LEAR [48]. Given the wealth of near-threshold data on $\bar{p} p \rightarrow \bar{\Lambda} \Lambda$, we expect that the interaction in the $\bar{\Lambda} \Lambda$ channel is fairly well constrained. The situation is much less satisfactory for other $\bar{Y} Y$ channels like $\bar{\Sigma}^{0} \Lambda$ and/or $\bar{\Sigma} \Sigma$ and, in particular, for $\bar{\Xi} \Xi$ where the $\bar{p} p \rightarrow \bar{Y} Y$ data base is rather poor.

Results for the energy dependence of the reaction cross sections and the effective form factors have been presented. Predictions are provided for the electromagnetic form factors $G_{M}$ and $G_{E}$ in the timelike region for the $\Lambda$, $\Sigma$, and $\Xi$ hyperons. It is found that the energy dependence of the near-threshold $e^{+} e^{-} \rightarrow \bar{\Lambda} \Lambda$ and $e^{+} e^{-} \rightarrow \bar{\Sigma}^{0} \Lambda$ cross sections reported by the BaBar collaboration [12] is well reproduced when employing various $\bar{\Lambda} \Lambda$ and $\bar{\Sigma}^{0} \Lambda$ potentials from 
52, 55 as final-state interaction. Both cross sections exhibit a sharp rise from the threshold and then remain basically constant for the next $100 \mathrm{MeV}$ or so, a feature that has been observed also in case of the reactions $e^{+} e^{-} \rightarrow \bar{p} p[\underline{3}$, 4,,$\underline{36}]$ and $e^{+} e^{-} \rightarrow \bar{n} n$ [5, 59]. This behavior of the cross sections implies an enhancement of the effective form factor for energies close to the threshold, a property which is likewise reproduced by the employed FSI. In case of $e^{+} e^{-} \rightarrow \bar{\Lambda} \Lambda$ even more delicate observables like the ratio $\left|G_{E} / G_{M}\right|$ and the relative phase between $G_{E}$ and $G_{M}$ can be described - at least at the energy of $2.396 \mathrm{GeV}$ where the, so far, only experimental information is available.

In view of the fact that there is only rather limited information on the $\bar{p} p \rightarrow \bar{\Sigma} \Sigma$ reaction, we have to acknowledge that the interaction utilized for the $\bar{\Sigma} \Sigma$ channel is much less reliable. Thus, the pertinent results reported in the present work have primarily an exploratory character. Nonetheless, it turned out that the energy dependence of the three channels $e^{+} e^{-} \rightarrow \bar{\Sigma}^{-} \Sigma^{+}, \bar{\Sigma}^{0} \Sigma^{0}, \bar{\Sigma}^{+} \Sigma^{-}$at low energies can be roughly reproduced. Our calculation suggests that there is a strong interplay between the $\bar{\Sigma}^{-} \Sigma^{+}, \bar{\Sigma}^{0} \Sigma^{0}$, and $\bar{\Sigma}^{+} \Sigma^{-}$channels in the near-threshold region, caused by the $\bar{\Sigma} \Sigma$ interaction. Therefore, it would be very interesting to perform further experiments that establish reliably the energy dependence of the cross sections for all three channels at low energies, but also those of other observables like the ratio $\left|G_{E} / G_{M}\right|$. The latter seems to be very sensitive to the details of the $\bar{Y} Y$ interaction and, thus, more extended measurements in the region of the $\bar{\Sigma} \Sigma$ thresholds would definitely provide a deeper insight into the $\bar{Y} Y$ dynamics.

Finally, let us mention that an alternative and independent source of information on the $\bar{Y} Y$ interaction is provided by studies of two-particle momentum correlation functions in heavy ion collisions and/or high-energy $p p$ collisions. Pertinent data on $\bar{\Lambda} \Lambda$ correlations have been already published by the ALICE Collaboration [43]. Extending those studies to further strange baryon-antibaryon interactions such as $\bar{\Sigma} \Sigma$ and/or $\Xi \Xi$ should be feasible. In addition there are plans by the $\bar{P}$ ANDA Collaboration at FAIR to resume the measurements of $\bar{p} p \rightarrow \bar{\Lambda} \Lambda, \bar{p} p \rightarrow \bar{\Sigma}^{0} \Lambda, \bar{p} p \rightarrow \bar{\Xi} \Xi$ 60, 61] that stopped when the LEAR facility at CERN was shut down. Further options to study the $\bar{Y} Y$ interaction as offered for example by reactions like $B \rightarrow \bar{\Lambda} \Lambda K$ [62] or $B \rightarrow \bar{\Lambda} \Lambda D$ [63] could be also exploited eventually.

\section{ACKNOWLEDGMENTS}

This work is supported in part by the Deutsche Forschungsgemeinschaft (DFG) and the National Natural Science Foundation of China (NSFC) through funds provided to the Sino-German CRC 110 "Symmetries and the Emergence of Structure in QCD". The work of UGM was also supported by the Chinese Academy of Sciences (CAS) President's International Fellowship Initiative (PIFI) (Grant No. 2018DM0034) and by the EU (Strong2020). L.-Y. Dai is supported by the NSFC with Grant No. 11805059, the Joint Large Scale Scientific Facility Funds of the NSFC and CAS under Contract No. U1932110, and Fundamental Research Funds for the Central Universities.

[1] G. Bardin et al., Nucl. Phys B 411, 3 (1994).

[2] B. Singh et al. [PANDA Collaboration], Eur. Phys. J. A 52, 325 (2016)

[3] B. Aubert et al., Phys. Rev. D 73, 012005 (2006).

[4] J.P. Lees et al., Phys. Rev. D 87, 092005 (2013).

[5] M. N. Achasov et al., Phys. Rev. D 90, 112007 (2014).

[6] M. Ablikim et al. [BESIII Collaboration], Phys. Rev. D 91, 112004 (2015).

[7] R. R. Akhmetshin et al. [CMD-3 Collaboration], Phys. Lett. B 759, 634 (2016).

[8] R. R. Akhmetshin et al. [CMD-3], Phys. Lett. B 794, 64 (2019).

[9] M. Ablikim et al. [BESIII], Phys. Rev. Lett. 124, 042001 (2020).

[10] A. Denig and G. Salmè, Prog. Part. Nucl. Phys. 68, 113 (2013).

[11] D. Bisello et al. [DM2 Collaboration], Z. Phys. C 48, 23 (1990).

[12] B. Aubert et al. [BaBar Collaboration], Phys. Rev. D 76, 092006 (2007).

[13] R. Baldini, S. Pacetti, A. Zallo and A. Zichichi, Eur. Phys. J. A 39, 315 (2009).

[14] O. D. Dalkarov, P. A. Khakhulin and A. Y. Voronin, Nucl. Phys. A 833, 104 (2010).

[15] G. Fäldt, Eur. Phys. J. A 51, 74 (2015).

[16] S. Dobbs, A. Tomaradze, T. Xiao, K. K. Seth and G. Bonvicini, Phys. Lett. B 739, 90 (2014).

[17] S. Dobbs, K. K. Seth, A. Tomaradze, T. Xiao and G. Bonvicini, Phys. Rev. D 96, 092004 (2017).

[18] M. Niiyama et al. [Belle], Phys. Rev. D 97, 072005 (2018).

[19] M. Ablikim et al. [BESIII], Phys. Rev. D 97, 032013 (2018).

[20] M. Ablikim et al. [BESIII], Phys. Rev. Lett. 123, 122003 (2019).

[21] M. Ablikim et al. [BESIII], arXiv:2009.01404 [hep-ex]].

[22] M. Ablikim et al. [BESIII], Phys. Rev. Lett. 124, 032002 (2020).

[23] M. Ablikim et al. [BESIII], arXiv:2010.08320 [hep-ex]]. 
[24] J. Haidenbauer and U.-G. Meißner, Phys. Lett. B 761, 456 (2016).

[25] X. Cao, J.-P. Dai and Y.-P. Xie, Phys. Rev. D 98, 094006 (2018).

[26] Y. Yang and Z. Lu, Mod. Phys. Lett. A 33, 1850133 (2018).

[27] Y. Yang, D.-Y. Chen and Z. Lu, Phys. Rev. D 100, 073007 (2019).

[28] L.-Y. Xiao, X.-Z. Weng, X.-H. Zhong and S.-L. Zhu, Chin. Phys. C 43, 113105 (2019).

[29] S. Dubnička, A. Z. Dubničkova, C. Adamuščin and E. Bartoš, PoS EPS-HEP2017, 757 (2018).

[30] G. Fäldt and A. Kupsc, Phys. Lett. B 772, 16 (2017).

[31] E. Perotti, G. Fäldt, A. Kupsc, S. Leupold and J. J. Song, Phys. Rev. D 99, 056008 (2019).

[32] R. Baldini-Ferroli, A. Mangoni and S. Pacetti, Eur. Phys. J. C 80, 903 (2020).

[33] G. Ramalho, M. T. Peña and K. Tsushima, Phys. Rev. D 101, 014014 (2020).

[34] G. Fäldt, Eur. Phys. J. A 52, 141 (2016).

[35] M. Ablikim et al. [BESIII], Chin. Phys. C 44, 040001 (2020).

[36] J. Haidenbauer, X.-W. Kang and U.-G. Meißner, Nucl. Phys. A 929, 102 (2014).

[37] X.-W. Kang, J. Haidenbauer and U.-G. Meißner, JHEP 1402, 113 (2014).

[38] L.-Y. Dai, J. Haidenbauer and U.-G. Meißner, JHEP 07, 078 (2017).

[39] D. Zhou and R. G. E. Timmermans, Phys. Rev. C 86, 044003 (2012).

[40] G. Pakhlova et al. [Belle Collaboration], Phys. Rev. Lett. 101, 172001 (2008).

[41] M. Ablikim et al. [BESIII], Phys. Rev. Lett. 120, 132001 (2018).

[42] L.-Y. Dai, J. Haidenbauer and U.-G. Meißner, Phys. Rev. D 96, 116001 (2017).

[43] S. Acharya et al. [ALICE], Phys. Lett. B 802, 135223 (2020).

[44] P.D. Barnes et al., Phys. Lett. B 229, 432 (1989).

[45] P.D. Barnes et al., Nucl. Phys. A 526, 575 (1991).

[46] P.D. Barnes et al., Phys. Rev. C 54, 1877 (1996).

[47] P.D. Barnes et al., Phys. Rev. C 62, 055203 (2000).

[48] E. Klempt, F. Bradamante, A. Martin and J.-M. Richard, Phys. Rept. 368, 119 (2002).

[49] P. D. Barnes et al. [PS185], Phys. Lett. B 402, 227 (1997).

[50] T. Johansson, Nucl. Phys. A 655, 173 (1999)

[51] T. Johansson, private communication.

[52] J. Haidenbauer, T. Hippchen, K. Holinde, B. Holzenkamp, V. Mull and J. Speth, Phys. Rev. C 45, 931 (1992).

[53] J. Haidenbauer, K. Holinde, V. Mull and J. Speth, Phys. Lett. B 291, 223 (1992).

[54] J. Haidenbauer, K. Holinde, V. Mull and J. Speth, Phys. Rev. C 46, 2158 (1992).

[55] J. Haidenbauer, K. Holinde and J. Speth, Nucl. Phys. A 562, 317 (1993).

[56] J. Haidenbauer, K. Holinde and J. Speth, Phys. Rev. C 47, 2982 (1993).

[57] J. Haidenbauer, K. Holinde and M. B. Johnson, Phys. Rev. C 45, 2055 (1992).

[58] B. Holzenkamp, K. Holinde and J. Speth, Nucl. Phys. A 500, 485 (1989).

[59] J. Haidenbauer, C. Hanhart, X.-W. Kang and U.-G. Meißner, Phys. Rev. D 92, 054032 (2015).

[60] K. Schönning, EPJ Web Conf. 241, 01015 (2020).

[61] G. Barucca et al. [PANDA], arXiv:2009.11582 [hep-ex]].

[62] Y. W. Chang et al. [Belle], Phys. Rev. D 79, 052006 (2009).

[63] J. P. Lees et al. [BaBar], Phys. Rev. D 89, 112002 (2014). 